\title{
Application Method of Landscape Elements in Interior Design
}

\author{
Xiaoling Zhu ${ }^{1}$ \\ ${ }^{1}$ Hebei Academy of Fine Arts, Xinle, Hebei, 050700
}

\section{KEYWORDS: Landscape Decoration; Interior Design; Applications}

\begin{abstract}
In today's rapid economic development, people's living environment has ever-increasing demands. As an independent subject of design, indoor environmental design can improve and increase the interior design superiority and level have played a significant role in promoting. It is not only an integral part of art and design, as well as a collection of cultural and historical, and artistic innovation as one of modern design category, while the landscape of one of the decorative elements is also a cultural and natural landscape design combines innovation mode. The use of interior design in the improvement of people's quality of life, work environment and enrich people have played a very big role. In this paper, through the use of decorative elements of landscape design in the indoor environment and the role to be analyzed in order to illustrate the relationship between the two, and the ways it should apply.
\end{abstract}

\section{Introduction}

With the continuous improvement of material conditions, people living in the city and ultimately will be in the fast-paced, high efficiency, stressful environment, so correspondingly there are more and more people in the pursuit of spiritual needs and satisfaction, resulting in progressively more and more people tend to love or nature style. The natural landscape decorative element refers to pro-scientific value and aesthetics, nature tour with ornamental scenery scene, and by the impact of human activities rarely a natural complex. Now that serious pollution, the environment is severely damaged, so the modern interior design, landscape decorative elements will be more and more loved by the people, it will also be a future trend in domestic interior design.

\section{The Basic Elements of Landscape Elements}

Eye clinics we can see the landscape are visible natural landscape belongs to the complex nature is a self-contained, decorative landscape elements were designed to process with a decorative and artistic value. To a small grass, large mountain is a manifestation of this element. It is the biggest difference between the natural landscape and cultural landscape is shaped by natural elements from, cultural landscape is often based on the natural landscape of the sitting of artificial modification. Thus, the more natural landscape that has a natural taste, people have some kind of immersive feel comfortable. Nature itself has given object can be personalized and spiritual characteristics of these apply to interior design or environmental design can enrich people's visual effects, but also improve its aesthetic value.

Modern society in the natural landscape design and more natural style building is characteristic of both traditional Chinese culture and China combined together to create the design concept, this design is not divorced from life and reality, but in the back to nature when stressed , but also to experience the real meaning of existence and value of life. Thus, the interior design of this design 
with landscape elements of nature has become the designers to achieve the ideal of the pursuit of their own environment.

As we all know the basic elements of nature refers to the elements of nature, mountains, water, earth, wood and sound, light, color and other natural formation. The man managed to create a style element that is naturally focus on the performance of such a natural, free, comfortable and harmonious combination of taste. In the natural style, in order to closer to the original ecosystem, it will allow the existence of rough and broken, and the materials used are unavoidable tend to respect for the natural elements, such as: stone, wood, bricks, pottery, bamboo, rattan and so is the best designers in the eyes of the material of choice. On braid like to choose some natural products like cotton because the texture of these materials and not decorated with carved rustic match. Sometimes designers in order to better reflect the natural elements or the use of space, will be designed as a living space nature green space, and sometimes will use a natural sense of style and natural fabrics to itself with good rendering countryside atmosphere, giving the play a real natural style lounge atmosphere.

\section{Relationship between Environmental Design and Landscape Elements}

The natural landscape elements are important and indispensable part of the interior design, the two are closely related. The indoor environment is a concentrated through a small space, but also people's lives and are inseparable from the place of residence, as a natural biological leader will be more human nature can not be separated, which is why everyone can not help but like big nature, close to nature and depend on a nature exists. Therefore, the natural landscape of this nature elements into the design of the indoor environment, not only to strengthen coexistence between man and nature in harmony, but also to meet people's needs and love of nature, the closer the relationship between people and nature.

Personalized, ideas of humanity and the natural landscape are all indispensable constituent elements, it has also been widely used in people's lives. Currently on the environment we live in, the learning and work and entertainment are inseparable from the existence of landscape decoration. For example, in the construction of small fresh style, the designer will be in the design process in the trees and plants in these natural features into the design, but also to join all kinds of hand-painted landscapes or other objects to bring out this natural style. So designers will often add a variety of bonsai, stones and flowers, birds and other elements, has reached reflect the true nature of this kind.

\section{The Application of Landscape Elements on Environmental Design}

With the progress of people's thinking, but also it will lead to the changing aesthetic, natural and will have a growing ability to accept new things. When the traditional design concept and design style can not meet the needs of the more modern society and people's spiritual pursuit, only to gradually strengthen and increase the landscape elements in interior design in use, in order to improve the modern environmental design required a comprehensive development of.

Humans are particularly small in the face of nature, so that people in the worship of nature at the same time there will be in awe side. Convey an aesthetic landscape elements of both the natural landscape to enjoy, with nature will give a moody impression. Aesthetic point of different people are various and different people's awareness and understanding of nature are not the same, which gives the main elements of landscape designers and more room for imagination. For example, some people like the warm cozy and romantic style, designers can use a combination of plants and warm 
light design concepts to meet customer requirements. Some people like the sleek style of the texture, the designer will be in the design process to the wood itself wood texture or color patterns as the main tone of the design. The total of these different design philosophy can be summarized as the natural landscape has the style to be able to adapt to the environment of the interior design.

In fact, the concept of an innovative application. We are currently used in indoor environment design up to the natural landscape elements can always bring back the most vivid sense: green, green, harmony, health, and popularity. With more and more serious environmental damage, people also began to chase the environmental awareness of energy conservation, this awareness is still growing, then follow from this is that environmental awareness has also been applied to the design of the environment, there is a phenomenon of the natural landscape of eco-friendly concept more widely used occur. Such eco-friendly landscape design not only meet the needs of consumers at heart, but also for the modern environmental protection has contributed to.

User-friendly application is from the perspective of human senses or, after compliance with the required standards of beauty and life of people, but also will get people's approval and love. Natural environment of human growth and life have a common space, but also the most familiar space, when the room is designed to penetrate the natural landscape elements later, you can give a very strong sense of belonging. The living environment, user-friendly design mostly reflected in many details. As people tend to be in the selection of health and environmental protection; for families with children who will love the simple and practical design, but also tend to swing like a favorite decoration. So many different types of landscape design, fully meet the different requirements at different levels at different stages of people.

In modern society people more and more passion to pursue different feeling, which is the personalization of the show. Indoor environment in space is limited, so naturally in the design is limited, especially in some of the resources and materials that can be used are limited in scope. However, the use of landscape elements in the interior design aspects is often good coverage anywhere, to construct unexpected results. In the eyes of designers, whenever there is a practical value and aesthetic value element exists, then they can become design materials used. As some people like to keep goldfish, the designer will use to design the glass floor to become, thereby creating a perspective effect of fish. Some people prefer a minimalist style or original style, designers will be added and some other plant natural green element in the living room, there will be a park-like feel Language occupants sitting under a tree when leisure time. The fact, that is personalized performance.

Now many public spaces are decorated with light interior spaces, the use of light to highlight the beauty of different visual effects on the well-being of light and shade or the like, the light can be increased three-dimensional visual, if not decorative light, it means there is no shape, color and space. Therefore, many designers love to use light to visualize the effect of the indoor environment design. For example, when the sun through large glass will produce future will enjoy plenty of sunshine and outdoor introduce natural scenery unreserved interior space, thereby forming a variety of patterns and color lines, resulting in the indoor environment become quite layered and colorful light. But also according to the principle of light refraction, so indoor display furniture also reflects the rich luster, so that the indoor environment becomes more natural and comfortable. Many designers will use in public buildings or residential environment and interior artificial lighting interface shape combine in the design, but with respect to artificial lighting, the reference to natural light to construct the indoor environment design not only become more clever, the effect is more natural. 
In the indoor environment design, if only to rely on a simple architectural elements to decorate the indoor environment, it will make the space seem monotonous level, rigid, boring, lifeless nor vivid. If these architectural elements can be added in some of the natural scenic elements, not only allows the active space, but also make the entire space becomes more and more vivid and rich. Appropriate viewing simulate natural sound of some elements of nature, it will make the rigid calm interior life and become more humane. Such as: wind, waves, the sound of a waterfall or a slight effect dynamic environment like the flowers, adding these elements not only make people have a sense of comfortable share of natural Language, but also in the invisible reduction work pressure.

Color in Interior Design has been very important, through the rational use of color can create the desired style and style to bring the art visual enjoyment. Therefore, we have been supported by the observation of natural elements into the design of color, into life. In the rapid development of today, the world is also due to color and more colorful, but no matter how developed we are still inseparable from inspiration to us by nature and beauty, but also inseparable from this space to us by nature. We advocate a return to nature, longing for nature, and nature is because we have always been associated with hard to break away. Great nature flowers and trees, rocks, water and soil are viewing the interior design elements of the best decorations, but also the best natural color. Well, as long as the use of the design of these natural colors, we can design a return to the real space and the most natural state, so that people living in this environment naturally felt a cordial and harmonious.

\section{Conclusion}

The above research can be a good description of landscape elements used throughout the indoor environment design and application of both the necessity and the importance of its existence. To improve and enhance the quality of the indoor environment design to be sure to maintain the integrity of the design patterns and innovative design concept, only the implementation of the genuineness of the reality of practice in order to more fully display their interior design aesthetic and its function value. At the same time, designers want to design a practical and aesthetic two factors co-exist good works, it is necessary to break through the old, usual obstacles in the traditional design based on the premise of continuous innovation and development of new thinking to go the road will really achieve landscape elements and interior design as one seamless, thus more lay a solid foundation for the sustainable development of indoor environments.

\section{REFERENCE:}

[1] Zhang Qingping, Interpreting the Development of the 20th Century Chinese interior design [J]. Nanjing Forestry University, 2004.

[2] Liu Pingping, Application landscape elements in interior design in the [J]. Zhejiang Agriculture and Forestry Science, 2014 (5).

[3] Hu Dayong., on the interior design inherit and carry forward the traditional culture of [J]. decoration, 2005 (5).

[4] Chen Yongchang. masterpieces Square - light, color, materials [M]. Beijing Interior Design, 2004 (3).

[5] Ren Xuequn, Liu Yanlin. landscape design elements in the interior design of [J]. Kehai stories Expo, Discovery Science and Technology, 2011 (1). 